\title{
Nucleon form factors and moments of parton distributions in twisted mass lattice QCD
}

\author{
C. Alexandrou ${ }^{(a, b)}$, M. Constantinou* ${ }^{(a)}$, C. Kallidonis ${ }^{(a)}$, T. Korzec ${ }^{(a, c)}$ \\ (a) Department of Physics, University of Cyprus, P.O. Box 20537, 1678 Nicosia, Cyprus \\ (b) Computation-based Science and Technology Research Center, Cyprus Institute, 20 Kavafi \\ Str., Nicosia 2121, Cyprus \\ (c) Institut für Physik Humboldt Universität zu Berlin, Newtonstrasse 15, 12489 Berlin, Germany \\ E-mail: alexand@ucy.ac.cy, constantinou.martha@ucy.ac.cy, \\ kallidonis.christos@ucy.ac.cy, korzec@physik.hu-berlin.de
}

\section{Brinet, J. Carbonell, P. A. Harraud, M. Papinutto}

Laboratoire de Physique Subatomique et Cosmologie, UJF/CNRS/IN2P3, 53 avenue des Martyrs, 38026 Grenoble, France

E-mail:marianedlpsc.in2p3.fr, Jaume.Carbonellelpsc.in2p3.fr, harraudelpsc.in2p3.fr, Mauro.Papinutto@lpsc.in2p3.fr

\section{P. Guichon}

CEA-Saclay, IRFU/SPhN, 91191 Gif-sur-Yvette, France

E-mail: pierre.guichonecea.fr

\section{K. Jansen}

NIC, DESY, Platanenallee 6, D-15738 Zeuthen, Germany

E-mail: Karl.Jansen@desy.de

\begin{abstract}
We present results on the electroweak form factors and on the lower moments of parton distributions of the nucleon, within lattice QCD using two dynamical flavors of degenerate twisted mass fermions. Results are obtained on lattices with three different values of the lattice spacings, namely $a=0.089 \mathrm{fm}, a=0.070 \mathrm{fm}$ and $\mathrm{a}=0.056 \mathrm{fm}$, allowing the investigation of cut-off effects. The volume dependence is examined by comparing results on two lattices of spatial length $\mathrm{L}=2.1 \mathrm{fm}$ and $\mathrm{L}=2.8 \mathrm{fm}$. The simulations span pion masses in the range of 260-470 MeV. Our results are renormalized non-perturbatively and the values are given in the MS-scheme at a scale $\mu=2 \mathrm{GeV}$.
\end{abstract}

XXIst International Europhysics Conference on High Energy Physics

21-27 July 2011

Grenoble, Rhône-Alpes France

\footnotetext{
* Speaker.
} 


\section{Introduction}

Lattice QCD calculations of observables related to the structure of baryons are now being carried out using simulations of the theory with parameters close to the physical regime both in terms of pion mass and with respect to the continuum limit $[1,2,3,4,5]$. In particular, a number of major collaborations are investigating the form factors and the first moments of parton distributions of the nucleon that encode important information on its structure. In this work we consider the nucleon matrix elements of the operators $\mathscr{O}_{V}^{\mu_{1} \ldots \mu_{n}}=\bar{\psi} \gamma^{\left\{\mu_{1}\right.} i \stackrel{\leftrightarrow}{D} \mu_{2} \ldots i \overleftrightarrow{D}{\left.\stackrel{\leftrightarrow}{\mu_{n}}\right\}} \psi$ and

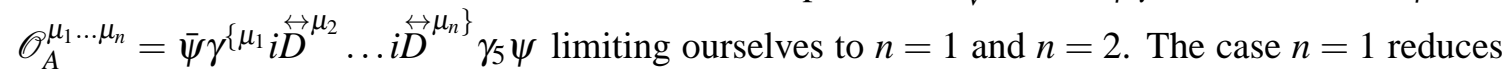
to the nucleon form factors. The nucleon matrix element of the electromagnetic current is written in the form

$$
\left\langle N\left(p^{\prime}, s^{\prime}\right)\left|j^{\mu}\right| N(p, s)\right\rangle=\left(\frac{m_{N}^{2}}{E_{N}\left(\mathbf{p}^{\prime}\right) E_{N}(\mathbf{p})}\right)^{1 / 2} \bar{u}_{N}\left(p^{\prime}, s^{\prime}\right)\left[\gamma^{\mu} F_{1}\left(q^{2}\right)+\frac{i \sigma^{\mu v} q_{v}}{2 m_{N}} F_{2}\left(q^{2}\right)\right] u_{N}(p, s),
$$

where $q^{2}=\left(p^{\prime}-p\right)^{2}, m_{N}$ is the nucleon mass and $E_{N}(\mathbf{p})$ its energy. $F_{1}(0)=1$ for the proton and zero for the neutron since we have a conserved current. $F_{2}(0)$ measures the anomalous magnetic moment. They are connected to the electric, $G_{E}$, and magnetic, $G_{M}$, Sachs form factors by the relations: $G_{E}\left(q^{2}\right)=F_{1}\left(q^{2}\right)+\frac{q^{2}}{\left(2 m_{N}\right)^{2}} F_{2}\left(q^{2}\right)$ and $G_{M}\left(q^{2}\right)=F_{1}\left(q^{2}\right)+F_{2}\left(q^{2}\right)$. Similarly the matrix element for the axial-vector current is given by

$$
\left\langle N\left(p^{\prime}, s^{\prime}\right)\left|A_{\mu}^{3}\right| N(p, s)\right\rangle=i\left(\frac{m_{N}^{2}}{E_{N}\left(\mathbf{p}^{\prime}\right) E_{N}(\mathbf{p})}\right)^{1 / 2} \bar{u}_{N}\left(p^{\prime}, s^{\prime}\right)\left[G_{A}\left(q^{2}\right) \gamma_{\mu} \gamma_{5}+\frac{q_{\mu} \gamma_{5}}{2 m_{N}} G_{p}\left(q^{2}\right)\right] \frac{1}{2} u_{N}(p, s) .
$$

We also study matrix elements of operators with a single derivative $(n=2)$. The matrix elements of these operators are parameterized in terms of the generalized form factors $A_{20}\left(Q^{2}\right), B_{20}\left(Q^{2}\right), C_{20}\left(Q^{2}\right)$ and $\tilde{A}_{20}\left(Q^{2}\right), \tilde{B}_{20}\left(Q^{2}\right)\left(Q^{2}=-q^{2}\right)$. The results are non-perturbatively renormalized [6]. In this work we use simulations with $N_{F}=2$ twisted mass fermions (TMF). They provide an attractive formulation of lattice QCD that allows for automatic $\mathscr{O}(a)$ improvement, infrared regularization of small eigenvalues and fast dynamical simulations [7]. For the calculation of the nucleon observables discussed in this work the automatic $\mathscr{O}(a)$ improvement is particularly relevant since it is achieved by tuning only one parameter in the action, requiring no further improvements on the operator level. Details on the lattice evaluation can be found in Refs. [5].

\section{Nucleon form factors}

Our lattice results on the nucleon axial charge, $g_{A}$ are shown in Fig. 1. We observe that results at our three different lattice spacings are within error bars and that results at the two different volumes are also consistent. In the same figure we compare to the results obtained using $N_{F}=$ $2+1$ domain wall fermions (DWF) by the RBC-UKQCD collaborations [4] and using a hybrid action with $2+1$ flavors of staggered sea and domain wall valence fermions by LHPC [8]. There is agreement among lattice results using different lattice actions even before taking the continuum and infinite volume limit indicating that lattice artifacts are small. The pion mass dependence for the nucleon axial charge has been studied within $\mathrm{HB} \chi \mathrm{PT}$ in the SSE formulation [9]. Fitting the volume corrected and continuum extrapolated TMF results shown in Fig. 1 we find $g_{A}=1.12(8)$, while fitting the raw lattice results we obtained $g_{A}=1.08(8)$ confirming that cut-off and volume corrections have indeed a small effect. 

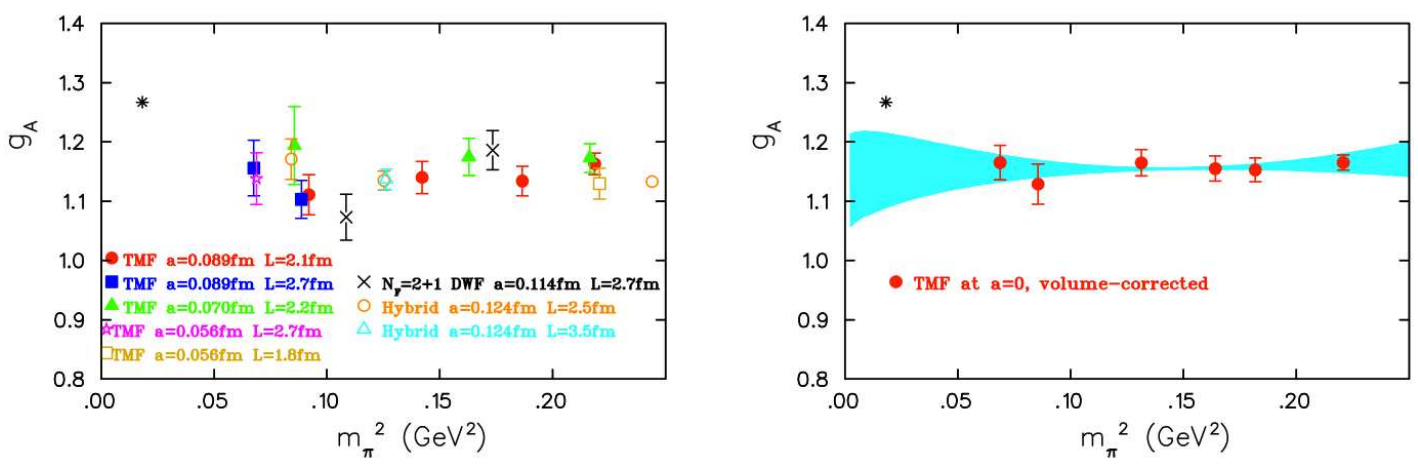

Figure 1: Left: Results on $g_{A}$ as a function of the pion mass squared using TMF, DWF and domain wall valence on a staggered sea (hybrid). Right: $g_{A}$ obtained by taking the continuum limit of the volume corrected TMF data. The shaded area is the best chiral fit to the data shown on the graph.
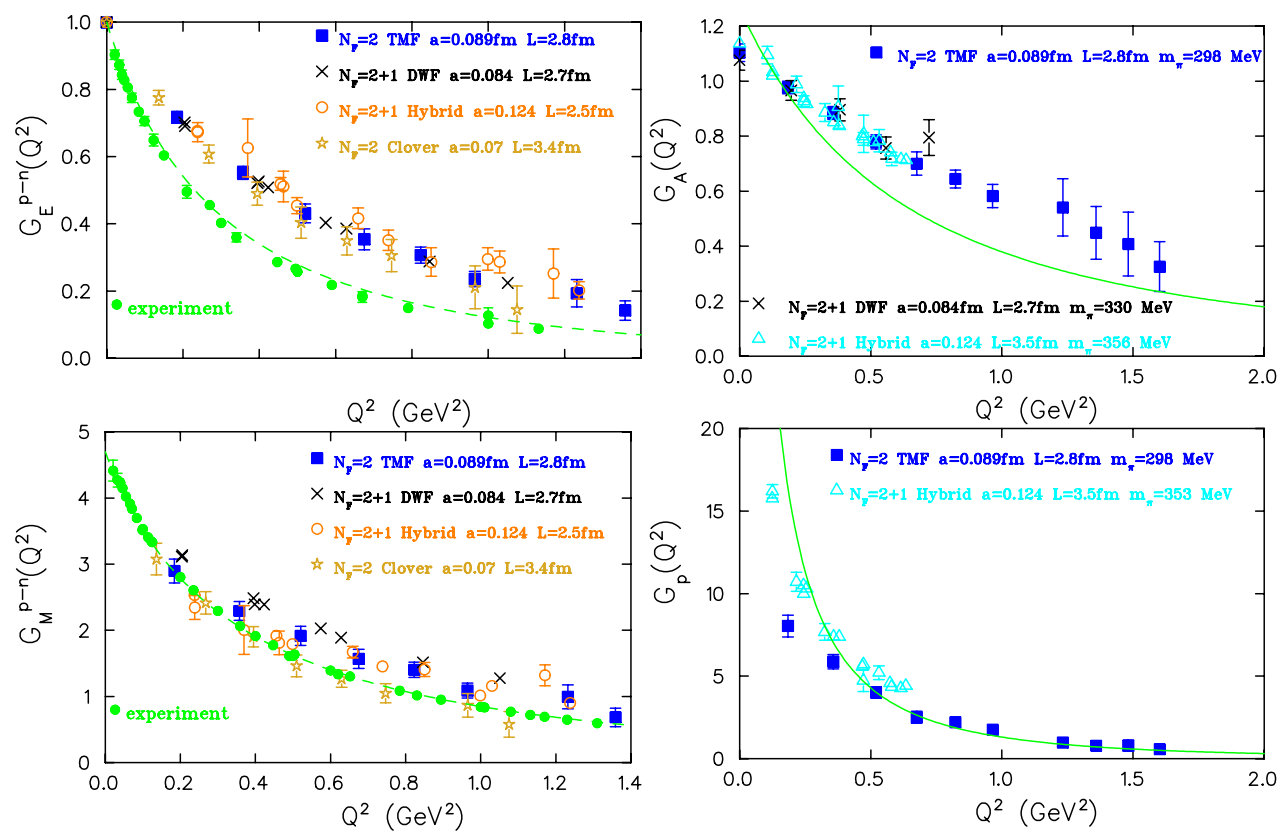

Figure 2: $G_{E}$ and $G_{M}$ at $m_{\pi} \sim 300 \mathrm{MeV}$ (left) and $G_{A}$ and $G_{p}$ (right) for various lattice actions. The filled (green) circles show experimental data on $G_{E}$ and $G_{M}$. The solid line on the right panel is a dipole fit to experimental data for $G_{A}\left(Q^{2}\right)$ combined with pion pole dominance to get the solid curve shown for $G_{p}\left(Q^{2}\right)$.

In Fig. 2 we show the isovector electric and magnetic form factors at $m_{\pi} \sim 300 \mathrm{MeV}$ as a function of the momentum transfer squared. We compare our results with results using other $\mathscr{O}(a)$ improved actions at the same pion mass $[8,10,11]$. The results are in agreement in the case of $G_{E}$, while in the case of $G_{M}$ clover results from Ref. [10] are lower. In the same figure we also show TMF results on the two axial-vector form factors and we compare with DWF at $m_{\pi}=330 \mathrm{MeV}$ [4] and hybrid results by LHPC at $m_{\pi}=356 \mathrm{MeV}$ [8] on a lattice with $L=3.5 \mathrm{fm}$. The results are in agreement in the case of $G_{A}\left(Q^{2}\right)$, while in the case of $G_{p}\left(Q^{2}\right)$ there are discrepancies at low $Q^{2}$ values, which may indicate that volume effects are not negligible on form factors such as $G_{p}\left(Q^{2}\right)$ which are strongly affected by the pion-pole. 

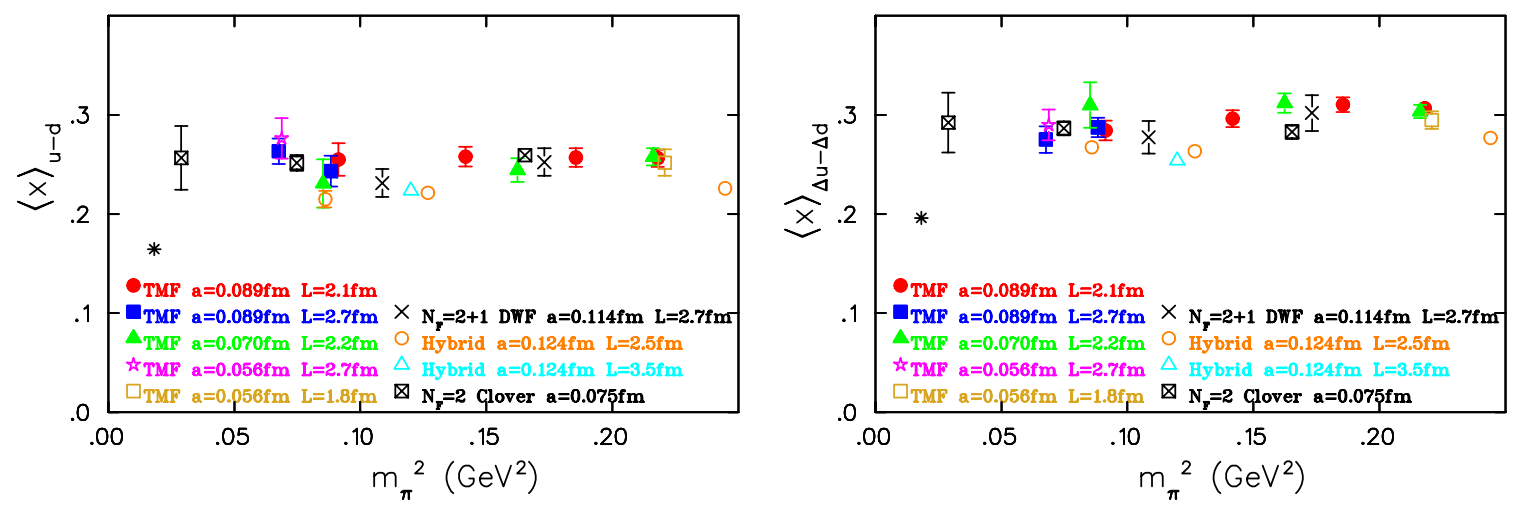

Figure 3: Our lattice data on $\langle x\rangle_{u-d}\left(A_{20}\right)$ and $\langle x\rangle_{\Delta u-\Delta d}\left(\tilde{A}_{20}\right)$ as a function of the pion mass squared. Results using other lattice actions are also plotted. The physical point is shown by the asterisk.

\section{Nucleon moments}

In Fig. 3 we show our results on the spin-independent and helicity moments, and results using other lattice actions $[8,12,13]$. Although to compare lattice data using different discretization schemes one would have to first extrapolate to the continuum limit, we find a good agreement among lattice results, due to small cut-off effects for lattice spacings of about $0.1 \mathrm{fm}$. Lattice values for $\langle x\rangle_{u-d}=A_{20}\left(Q^{2}=0\right)$ although compatible, are higher from the phenomenological value $\left\langle x_{u-d}\right\rangle \sim 0.16$. A similar conclusion holds for the helicity moment.

\section{Conclusions}

Using $N_{F}=2$ twisted mass fermions we obtain accurate results on the electromagnetic and axial form factors, as well as the lower moments of parton distributions of the nucleon. The results are obtained as a function of $Q^{2}$ for pion masses in the range of about $260-470 \mathrm{MeV}$. The general feature is a flatter dependence on $Q^{2}$ than experiment. Our results are in agreement with recent results obtained using other lattice fermions such as dynamical $N_{F}=2+1$ domain wall fermions. Having results at three lattice spacings enables us to take the continuum limit. We find that cutoff effects are small for the values of the lattice spacings used in this work. We also examine the pion mass dependence of the form factors as well as of the quantities derived by fitting the $Q^{2}$-dependence of these form factors.

Acknowledgments: This work was partly supported by funding received from the Cyprus Research Promotion Foundation under contracts EPYAN/0506/08, and TECHNOLOGY/OEПI $\Sigma / 0308(B E) / 17$. M. P. acknowledges financial support by a Marie Curie European Reintegration Grant of the 7th European Community Framework Programme under contract number PERG05-GA-2009-249309.

\section{References}

[1] P. Hagler et al. (LHPC) Phys. Rev. D77 (2008) 094502, arXiv:0705.4295.

[2] D. Brommel et al. (QCDSF-UKQCD), PoS LAT2007 (2007) 158, arXiv:0710.1534.

[3] C. Alexandrou, PoS LATTICE2010 (2010) 001, arXiv:1011.3660.

[4] T. Yamazaki et al., Phys. Rev. D79 (2009) 114505, arXiv:0904.2039. 
[5] C. Alexandrou et al. (ETMC), Phys.Rev. D83 (2011) 045010, arXiv:1012.0857; Phys. Rev. D83 (2011) 094502, arXiv:1102.2208; Phys. Rev. D83 (2011) 114513, arXiv:1104.1600.

[6] C. Alexandrou, M. Constantinou, T. Korzec, H. Panagopoulos, F. Stylianou Phys. Rev. D83 (2011) 014503, arXiv:1006.1920; PoS Lattice 2010 (2010) 224, arXiv:1012.2981.

[7] A. Shindler, Phys. Rept. 461 (2008) 37, arXiv:0707.4093.

[8] J. Bratt et al. (LHPC), Phys. Rev. D82 (2010) 094502, arXiv:1001.3620.

[9] T. Hemmert, M. Procura, W. Weise, Phys. Rev. D68 (2003) 075009, hep-lat/0303002.

[10] S. Capitani, B. Knippschild, M. Della Morte, H. Wittig, PoS(LATTICE2010) (2010) 147.

[11] S. Syritsyn et al., Phys. Rev. D81 (2010) 034507, arXiv:0907.4194.

[12] D. Pleiter et al. (QCDSF/UKQCD), PoS LATTICE2010 (2010) 153, arXiv:1101.2326.

[13] Y. Aoki et al., Phys. Rev. D82 (2010) 014501, arXiv:1003.3387. 